\title{
Identification of latent neosporosis in sheep in Tehran, Iran by polymerase chain reaction using primers specific for the $\mathrm{Nc}-5$ gene
}

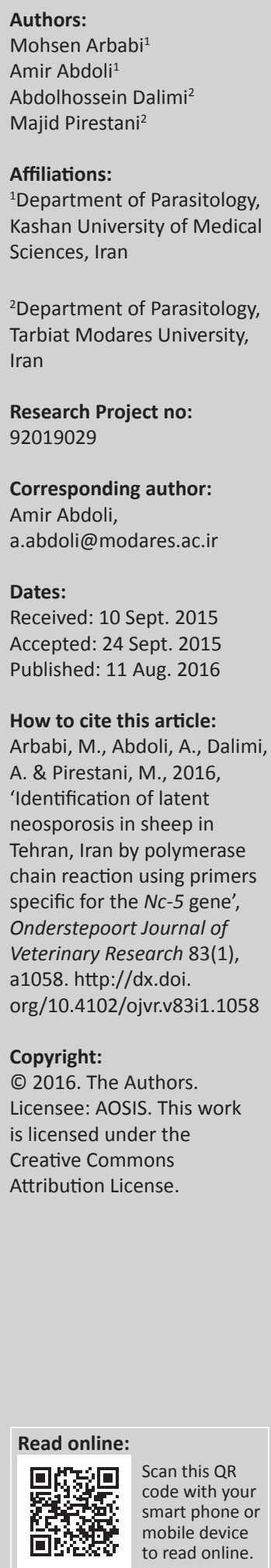

\begin{abstract}
Little is known about latent infection and molecular characterisation of Neospora caninum in sheep (Ovis aries). In this study, 330 sheep samples (180 hearts and 150 brains) were analysed for $N$. caninum DNA by nested polymerase chain reaction (PCR) targeting the Nc-5 gene. Neospora caninum DNA was detected in 3.9\% (13/330) of sheep samples. The parasite's DNA was detected in $6.7 \%$ of heart samples $(12 / 180)$ and $0.7 \%(1 / 150)$ of brain samples. No clinical signs were recorded from infected or uninfected animals. Sequencing of the genomic DNA revealed $96 \%-99 \%$ similarity with each other and $95.15 \%-100 \%$ similarity with $N$. caninum sequences deposited in GenBank. To our knowledge, this is the first report on the use of PCR to identify latent neosporosis in sheep in Iran. The results of this study have the potential to contribute to our understanding of the role of $N$. caninum-infected sheep in the epidemiology of neosporosis.
\end{abstract}

\section{Introduction}

Neospora caninum is a worldwide protozoan having a variety of animal hosts (Dubey \& Schares 2011; Dubey, Schares \& Ortega-Mora 2007). Domestic and wild canids are definitive, whereas different bird and mammalian species (such as cattle, water buffalo, and sheep) serve as intermediate hosts (Dubey \& Schares 2011). Abortion, especially in dairy cattle, is one of the major consequences of neosporosis in animal husbandry (Almeria \& López-Gatius 2013) that lead to significant economic losses (Reichel et al. 2013). Moreover, ovine abortion and reproductive failure due to neosporosis have been reported in several studies (Dubey \& Lindsay 1990; Howe et al. 2008, 2012; Jolley et al. 1999; Moreno et al. 2012; Pena et al. 2007). In different studies, antibodies to N. caninum have been detected in $1.1 \%-8.3 \%$ of sheep in the west of Iran (Ezatpour et al. 2015; Gharekhani \& Heidari 2014), 27.7\% in Pakistan (Nasir et al. 2012), 2.1\% in Turkey (Gökçe et al. 2015), 10.3\% in China (Liu et al. 2015), 16.8\% in Greece (Anastasia et al. 2013), 3\% in Argentina (Hecker et al. 2013), and 13.1\% in south-eastern Brazil (Da Silva Andrade et al. 2012). However, there is little information describing the detection of nucleic acids resulting from latent neosporosis in sheep.

Until now, different genes such as internal transcribed spacer sequences, 18S-like ribosomal DNA (small-subunit rDNA), and Nc-5 genes have been used for molecular diagnosis of neosporosis (reviewed by Al-Qassab, Reichel \& Ellis [2010]; Goodswen, Kennedy \& Ellis [2013]). However, studies have indicated that the Nc-5 gene is one of the most highly sensitive and specific for the detection of neosporosis (Almerra et al. 2002; Dubey et al. 2014; Hughes et al. 2006; Kaufmann et al. 1996; Paula et al. 2004; Yamage, Flechtner \& Gottstein 1996) because it is repeated in the N. caninum sequence (Al-Qassab et al. 2010). Hence, the main objective of this study was to investigate detection and molecular characterisation of latent neosporosis in sheep (Ovis aries) in Tehran, Iran, by polymerase chain reaction (PCR) using primers specific for the $\mathrm{Nc}-5$ gene.

\section{Materials and methods}

\section{Animals and study area}

A total of 330 samples from healthy slaughtered sheep (180 hearts and 150 brains) were purchased from an abattoir in Vavan (located in the vicinity of Tehran) from April to September 2014. The animals tested originated from different counties (Eslamshahr, Shahriar, Robatkarim), all of which are located between $50 \mathrm{~km}$ and $200 \mathrm{~km}$ from Tehran. These locations have hot summers and moderate winters. No clinical signs such as fever, lymphadenitis, nasal and ocular discharges, or jaundice were recorded in any of the animals before slaughter. 


\section{DNA extraction}

The whole brain and heart of each sheep were individually rinsed with distilled water, packaged, and refrigerated. Approximately $200 \mathrm{~g}-250 \mathrm{~g}$ of different segments of brain and heart were homogenised with a pestle and mortar in liquid nitrogen, and DNA was extracted using a phenolchloroform extraction method as described in our recent report (Abdoli et al. 2015). To prevent DNA crosscontamination, all materials that were used between different tissue samples were decontaminated with sodium hypochlorite solution (2.5\%) and rinsed with distilled water. The concentration of DNA was determined by NanoDrop spectrophotometer (Thermo Fisher Scientific, Wilmington, DE, USA) for each sample. Overall, the DNA concentration ranged between $150 \mathrm{ng} / \mu \mathrm{L}$ and $200 \mathrm{ng} / \mu \mathrm{L}$.

\section{Nested polymerase chain reaction}

Nested PCR was conducted using specific primers for the Nc-5 gene. The first round of PCR was conducted using a pair of N. caninum-specific primers, Np21plus (5'-CCCAGTGCGTCCAATCCTGTAAC-3') and Np6plus (5'-CTCGCCAGTCCAACCTACGTCTTCT-3') (Muller et al. 1996). Nested PCR was performed with the primers Np6 (5'-CAGTCAACCTACGTCTTCT-3') and Np7 (5'-GGGTGAACCGAGGGAGTTG-3') (Hughes et al. 2006). Each amplification was performed in $20-\mu \mathrm{L}$ reaction mixtures containing $10 \mu \mathrm{L}$ of $2 x$ master mixes (DFS Master Mix, BIORON GmbH, Ludwigshafen, Germany), each of the respective primers (10 pmol for the first round reaction and 25 pmol for nested PCR), $7 \mu \mathrm{L}$ of distilled water, and 1 $\mu \mathrm{L}$ of template DNA. One microlitre of the first round product was used as the template for nested PCR. For each reaction, a negative control (double distilled water) and a positive control (DNA extracted from the $N_{c}-5$ strain of $N$. caninum) were included. Amplification was performed with initial denaturation for 5 minutes at $94^{\circ} \mathrm{C}$, followed by 40 cycles at $94{ }^{\circ} \mathrm{C}$ for 40 seconds (denaturation), annealing at $62{ }^{\circ} \mathrm{C}$ in the first round, and $56{ }^{\circ} \mathrm{C}$ in nested PCR for 40 seconds, extension at $72{ }^{\circ} \mathrm{C}$ for 40 seconds, and final extension at $72{ }^{\circ} \mathrm{C}$ for 10 minutes. PCR products were electrophoresed on a $1.5 \%$ agarose gel stained with safe stain (Sinaclon, Tehran, Iran) and visualised under ultraviolet trans-illumination.

\section{Nucleotide sequence analysis}

Four positive PCR products (from the second reaction) were amplified with a master mix containing Pfu DNA polymerase (Thermo Fisher Scientific, Waltham, USA, cat. no. EP0501), extracted from the gel (Vivantis gel purification kit, Selangor Darul Ehsan, Malaysia) according to the manufacturer's protocols. Then the products were sequenced in the forward and reverse directions by Sequetech (Mountain View, CA, USA) (Abdoli et al. 2015). The sequences were edited with BioEdit sequence alignment editor (Hall 1999), aligned with Nc-5 partial sequences from other hosts by ClustalX2.12 (Larkin et al. 2007) and compared with sequences of N. caninum available in GenBank. Phylogenetic trees were inferred and evolutionary analyses were conducted using the Tamura three-parameter option of the neighbour-joining model with MEGA6 software (http:/ / www.megasoftware. net/) (Tamura et al. 2013). The bootstrap scores were calculated for 1000 replicates (Tamura et al. 2013).

\section{Results}

Neospora caninum DNA was detected in 13 out of 330 sheep samples (3.9\%). The infection rates in the heart and brain samples were $6.7 \%(12 / 180)$ and $0.7 \%(1 / 150)$, respectively. Four nucleotide sequences of the $N c-5$ gene with a length of $227 \mathrm{bp}$ (Figure 1) were submitted to the GenBank database (GenBank accession numbers KR106181, KR106182, KR106183, KR106184). The results demonstrated our sequences shared $96 \%$ - 99\% similarity with each other (Figures 2 and 3) and 95\% - 100\% similarity with N. caninum deposited in GenBank (Appendix Figure 1). Phylogenetic trees showed intraspecific variations between our isolates and other N. caninum specimens deposited in GenBank (Figure 2). Analysis of our sequences showed 96.9\% - 97.8\% similarity with N. caninum isolated from sheep (DQ077661) in the UK and $96.9 \%-99.1 \%$ similarity with $N$. caninum isolated from sparrows (Passer domesticus) in Iran. Interestingly, one of our samples (KR106181) showed 100\% similarity with $N$. caninum isolated from wolves (Canis lupus) (KF649846) in the United States.

\section{Discussion}

Although an association between ovine abortion and neosporosis has been reported in different studies (Dubey \& Lindsay 1990; Howe et al. 2008, 2012; Jolley et al. 1999; Moreno et al. 2012; Pena et al. 2007), there is little information describing molecular detection of latent neosporosis in sheep. Here, we found a total infection rate of 3.9\% (13/330) in our sheep samples. Interestingly, 12 out of 13 positive samples were detected in the hearts and one positive sample was diagnosed in the brain. In previous studies, the seroprevalence of $N$. caninum has been reported in a range of $1.1 \%-8.3 \%$ of sheep from the west of Iran (Ezatpour et al. 2015; Gharekhani \& Heidari 2014). Moreover, N. caninum DNA was detected in $8.5 \%$ (Asadpour et al. 2013) and $0.9 \%$ of aborted ovine fetuses

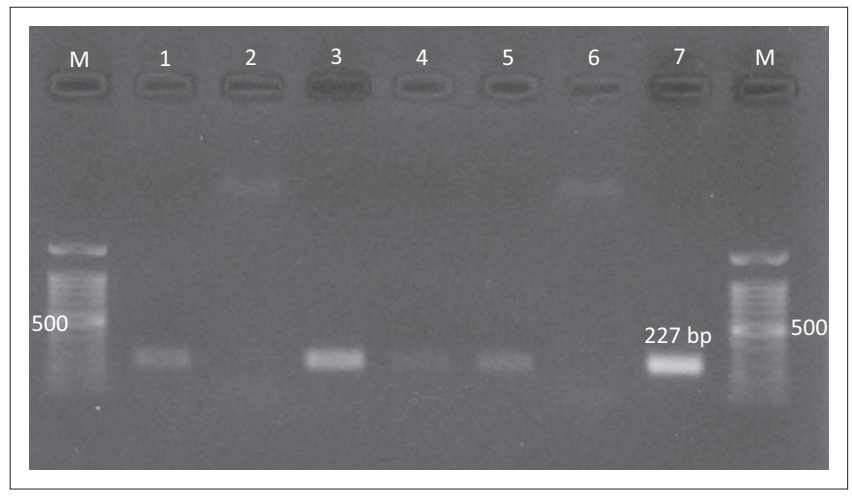

M, 100 bp DNA marker; Lane 1, positive control; Lane 2, negative control; Lanes 3, 4, 5, 7 , positive samples; Lane 6, negative samples.

FIGURE 1: Polymerase chain reaction products of four Neospora caninum positive samples. 


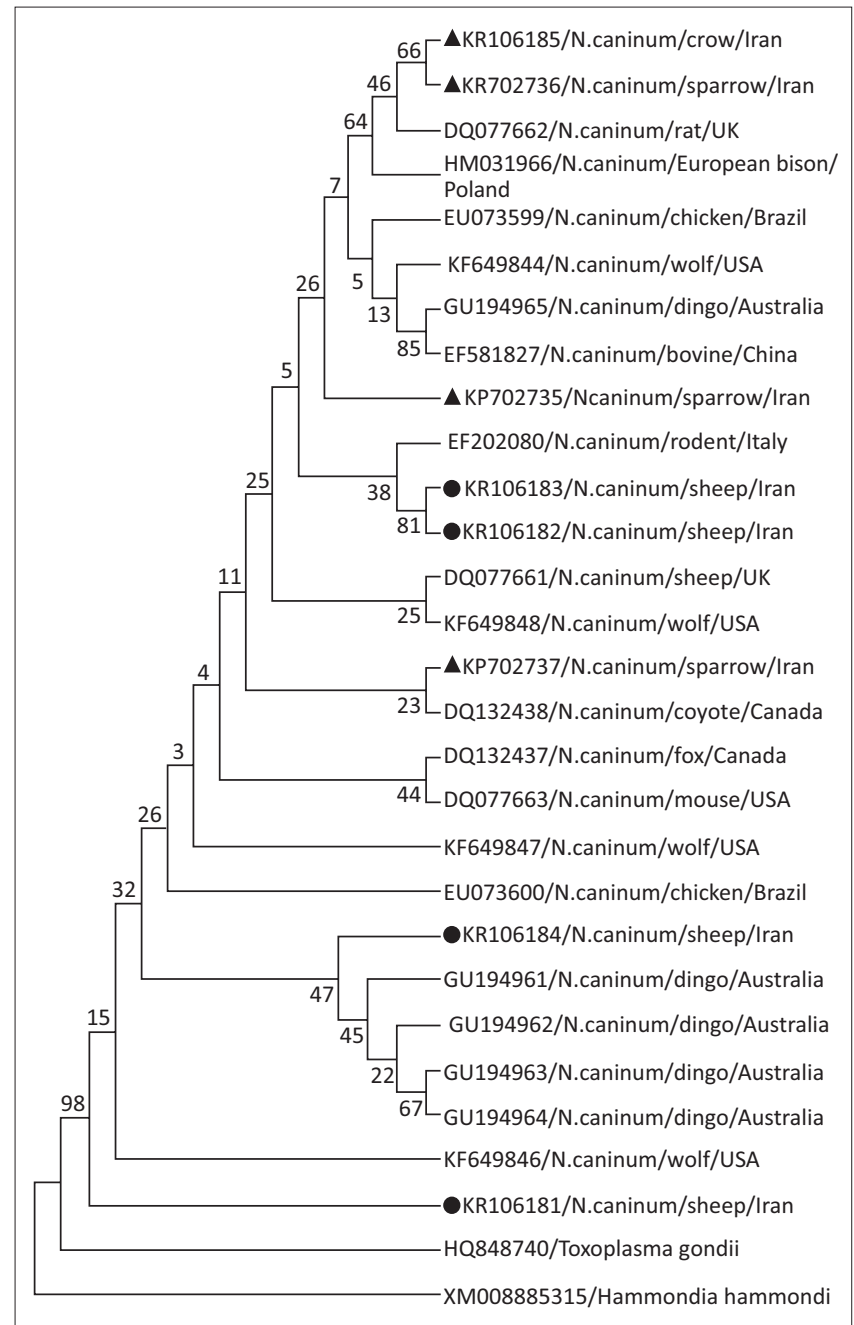

The evolutionary history was inferred using the neighbour-joining method. The branches are supported by 1000 bootstrap replicates.

- Neospora caninum isolated in the present study; $\boldsymbol{\Lambda}$, denotes Neospora caninum isolates in Iran.

FIGURE 2: Phylogenetic relationships among Neospora caninum specimens based on a fragment of the Nc-5 sequence. in Iran (Sasani et al. 2013). Şuteu et al. detected N. caninum DNA in the diaphragm tissues of 2 out of $181(1.1 \%)$ slaughtered goat kids in Romania (Şuteu et al. 2013). In the majority of studies, N. caninum was detected in brain samples from aborted or naturally infected sheep (Asadpour et al. 2013; Bishop et al. 2010; Dubey \& Lindsay 1990; Sasani et al. 2013; Silva et al. 2009). In this regard, Silva and colleagues detected N. caninum DNA in 2 out of 102 slaughtered goats $(1.92 \%)$ in Brazil. Interestingly, both positive samples were isolated from brain samples, whereas all heart and tongue samples were negative (Silva et al. 2009). Santos et al. (2010) detected N. caninum DNA in 5 out of 100 brain samples of beef cattle in Brazil, whereas none of the heart samples were positive (Santos et al. 2010). These results are dissimilar to our report, in which most of the positive samples were detected in the heart samples rather than in the brain samples $(6.7 \%$ versus $0.7 \%$ ). Our results also indicated that in sheep the heart is more susceptible to $N$. caninum infection than the brain.

Latent neosporosis can reactivate in conditions such as immunosuppression and pregnancy (Andrianarivo et al. 2005; Hemphill, Vonlaufen \& Naguleswaran 2006; Magaña et al. 2015; Mazuz et al. 2016; Pabón et al. 2007; Rettigner et al. 2004). Latently infected animals are also a source of $N$. caninum infection for canine definitive hosts.

In the current study, we used the Nc-5 gene for detection and phylogenetic analysis of N. caninum. This gene is repeated in the N. caninum sequence (Al-Qassab et al. 2010); hence, it is presented as a highly sensitive and specific gene for detection of neosporosis (Kaufmann et al. 1996; Yamage et al. 1996). The earlier study in this regard was conducted by Yamage et al. (1996), who compared the sensitivity and specificity of different primers for diagnosis of $N$. caninum. In this study, five forward (Np1, Np3, Np5, Np7, Np21) and four reverse (Np2, Np4, Np6, Np8) oligonucleotide primers that derived from the $\mathrm{Nc}_{-} 5$ genes were compared for the detection of

\footnotetext{
KR106181

$\mathrm{KR} 106184$

KR106182

KR106183

KR106181

KR106184

KR106182

KR106183

KR106181

KR106184

KR106182

KR106183

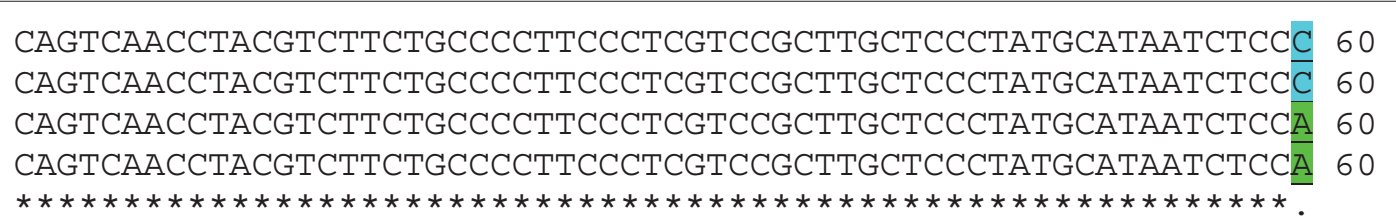

CCGTCATCAGCGCCGCCGGTGTTGCCTCAACACAGAACACTGAACTCTCGATAAGTATTA 120 CCGTCATCAGCGCCGCCGGTGTTGCCTCAACACAGAACACTGAACTCTCGATAAGTATCA 120 CCGTCATCAGTGCCGCCGGTGTTGCCTCAACACAGAACACTGAACTCTGGATAAGTATCA 120 GCGTCATCAGTGCCGCCGGTGTTGCCTCAACACAGAACACTGAACTCTGGATAAGTATCA 120

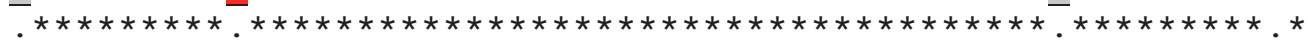

TTGGCACACTGTCCACACCCTGACGCAGGCTGATTTCAACGTGACGAATGACTAACCACA 180 TTGĀCACACTGTCCTCACCCTGACGCAGGCTG̈ATTTCAACGTGACGAATGACTAACCACA 180 TTGACACACTGTCCACACCCTGACGCAGGCTC̈ATGTCAACGTGACGAATGACTAACCACA 180 TTGACACACTGTCCACAGCCTGACGCAGGCTCATGTCAACGTGACGAATGACTAACCACA 180

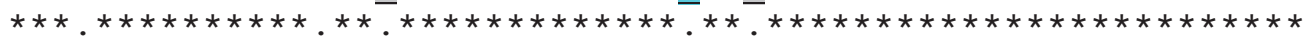

An asterisk represents an exact match between all sequences; a dot $(\bullet)$ represents a mismatch in at least one sequence.

FIGURE 3: Partial sequences of the Nc-5 gene from four isolates of Neospora caninum from sheep samples. 
neosporosis in experimentally infected mice. Among 19 combinations of forward and reverse primers, the Np21/ $\mathrm{Np6}, \mathrm{Np} 7 / \mathrm{Np} 6$, and Np2l/Np4 primer pairs were able to detect at least $10 \mathrm{pg}$ genomic DNA with a specific single band (Yamage et al. 1996). The Nc-5 gene can also discriminate $N$. caninum from other related apicomplexan parasites (Toxoplasma gondii and Sarcocystis species) (Kaufmann et al. 1996). Thus, the Nc-5 gene has been used as a highly sensitive and specific gene for detection of neosporosis (Almeria et al. 2002; Dubey et al. 2014; Hughes et al. 2006; Paula et al. 2004; Yamage et al. 1996). Hence, we selected the Nc-5 gene for sensitive and specific detection of neosporosis in the current study.

We also sequenced four positive samples for phylogenetic analysis. We found that our sequences displayed similarity levels of $96 \%-99 \%$ with each other (Figure 3) and 95\% $100 \%$ with $N$. caninum sequences deposited in GenBank (Appendix Figure 1). In comparison with molecular diagnosis, few studies have been conducted on the phylogenetic analysis of $N$. caninum with the Nc-5 gene (Auriemma et al. 2014; Čobádiová et al. 2013; Hughes et al. 2006). BLAST analyses indicated greater than $94 \%$ (Čobádiová et al. 2013), 96\% (Auriemma et al. 2014), and 97\% (Hughes et al. 2006) similarities between their sequences and other $N$. caninum sequences deposited in GenBank. It therefore seems that the Nc-5 gene is not a suitable biomarker for phylogenetic analysis and discrimination of genetic diversity for N. caninum. Instead, this gene is rather a highly sensitive and specific biomarker for the diagnosis of neosporosis. The use of ribosomal DNA, ITS-1, and recently microsatellites have been recommended for discriminating between N. caninum isolates (Al-Qassab et al. 2010).

Taken together, the results of this study provide molecular and epidemiological information about latent $N$. caninum infection in sheep in Iran. It can be expected that in future these results will contribute to revealing the role of latent $N$. caninum infection in the epidemiology of neosporosis in sheep.

\section{Acknowledgements}

This work was supported by the Iran National Science Foundation (grant no. 92019029). The authors would like to thank Mr. Mahdi Rezapour, Ali Karaji, and Ali Shafiei for their help in preparing the samples.

\section{Competing interests}

The authors declare that they have no financial or personal relationships that may have inappropriately influenced them in writing this article.

\section{Authors' contributions}

M.A. conceived the study design, analysed and interpreted the data and reviewed the manuscript; A.A. collected the data, prepared the samples, performed molecular assays, designed the tables and figures and wrote the manuscript. M.P. performed the molecular assays and sequencing analysis; A.D. conceived the study design and analysed and interpreted the data.

\section{References}

Abdoli, A., Arbabi, M., Dalimi, A. \& Pirestani, M., 2015, 'Molecular detection of Neospora caninum in house sparrow (Passer domesticus) in Iran', Avian Pathology 44(2), 319-322. http://dx.doi.org/10.1080/03079457.2015.1050583

Almerıa, S., Ferrer, D., Pabón, M., Castella, J. \& Manas, S., 2002, 'Red Foxes (Vulpes vulpes) are a natural intermediate host of Neospora caninum', Veterinary
Parasitology 107, 287-294. http://dx.doi.org/10.1016/S0304-4017(02)00162-0

Almeria, S. \& López-Gatius, F., 2013, 'Bovine neosporosis: Clinical and practical aspects', Research in Veterinary Science 95, 303-309. http://dx.doi.org/10.1016/j. rvsc.2013.04.008

Al-Qassab, S.E., Reichel, M.P. \& Ellis, J.T., 2010, 'On the biological and genetic diversity in Neospora caninum', Diversity 2, 411-438. http://dx.doi.org/10.3390/d2030411

Anastasia, D., Elias, P., Nikolaos, P., Charilaos, K. \& Nektarios, G., 2013, 'Toxoplasma gondii and Neospora caninum seroprevalence in dairy sheep and goats mixed stock farming', Veterinary Parasitology 198, 387-390. http://dx.doi.org/10.1016/j. vetpar.2013.09.017

Andrianarivo, A.G., Anderson, M.L., Rowe, J.D., Gardner, I.A., Reynolds, J.P., Choromanski, L. et al., 2005, 'Immune responses during pregnancy in heifers naturally infected with Neospora caninum with and without immunization', Parasitology Research 96, 24-31. http://dx.doi.org/10.1007/s00436-005-1313-y

Asadpour, R., Jafari-Joozani, R. \& Salehi, N., 2013, 'Detection of Neospora caninum in ovine abortion in Iran', Journal of Parasitic Diseases 37, 105-109.

Auriemma, C., Lucibelli, M.G., Borriello, G., De Carlo, E., Martucciello, A., Schiavo, L. et al., 2014, 'PCR detection of Neospora caninum in water Buffalo Foetal Tissues', Acta Parasitologica 59, 1-4. http://dx.doi.org/10.2478/s11686-014-0201-y

Bishop, S., King, J., Windsor, P., Reichel, M.P., Ellis, J. \& Šlapeta, J., 2010, 'The first report of ovine cerebral neosporosis and evaluation of Neospora caninum prevalence in sheep in New South Wales', Veterinary Parasitology 170, 137-142. http://dx.doi.org/10.1016/j.vetpar.2010.01.030

Čobádiová, A., Vichova, B., Majlathova, V. \& Reiterová, K., 2013, 'First molecular detection of Neospora caninum in European Brown Bear (Ursus arctos)', Veterinary Parasitology 197, 346-349. http://dx.doi.org/10.1016/j.vetpar.2013. 05.005

Da Silva Andrade, G., Bruhn, F.R.P., Rocha, C.M.B.M., De Sá Guimarães, A., Gouveia, A.M.G. \& Guimarães, A.M., 2012, 'Seroprevalence and risk factors for Neospora A.M.G. \& Guimarães, A.M., 2012, 'Seroprevalence and risk factors for Neospora
caninum in sheep in The State Minas Gerais, Southeastern Brazil', Veterinary caninum in sheep in The State Minas Gerais, Southeastern Brazil', Veterinar
Parasitology 188, 168-171. http://dx.doi.org/10.1016/j.vetpar.2012.03.006

Dubey, J., Jenkins, M., Ferreira, L., Choudhary, S., Verma, S., Kwok, O. et al., 2014, Isolation of viable Neospora caninum from brains of Wild Gray Wolves (Canis Iupus)', Veterinary Parasitology 201, 150-153. http://dx.doi.org/10.1016/j.vetpar. 2013.12.032

Dubey, J. \& Lindsay, D.S., 1990, 'Neospora caninum induced abortion in sheep', Journal of Veterinary Diagnostic Investigation 2, 230-233. http://dx.doi.org/10.1177/ 104063879000200316

Dubey, J. \& Schares, G., 2011, 'Neosporosis in animals - The last five years', Veterinary Parasitology 180, 90-108. http://dx.doi.org/10.1016/j.vetpar.2011.05.031

Dubey, J., Schares, G. \& Ortega-Mora, L., 2007, 'Epidemiology and control of Neosporosis and Neospora caninum', Clinical Microbiology Reviews 20, 323-367. http://dx.doi.org/10.1128/CMR.00031-06

Ezatpour, B., Alirezaei, M., Hassanvand, A., Zibaei, M., Azadpour, M. \& Ebrahimzadeh, F., 2015, 'The first report of Neospora caninum prevalence in aborted and healthy sheep from west of Iran', Comparative Clinical Pathology 24, 19-22. http://dx.doi. sheep from west of Iran', Comparati
org/10.1007/s00580-013-1846-x

Gharekhani, J. \& Heidari, H., 2014, 'Serology based comprehensive study of Neospora infection in domestic animals in Hamedan Province, Iran', Journal Advances Veterinary Animal Research 1, 119-124. http://dx.doi.org/10.5455/ javar.2014.a23

Gökçe, G., Mor, N., Kırmızıgul, A., Bozukluhan, K. \& Erkılıc, E., 2015, 'The first report of seropositivity for Neospora caninum in sheep from Turkey', Israel Journal of Veterinary Medicine 70, 40-44.

Goodswen, S.J., Kennedy, P.J. \& Ellis, J.T., 2013, 'A review of the infection, genetics, and evolution of Neospora caninum: From the past to the present', Infection, Genetic and Evolution 13, 133-150. http://dx.doi.org/10.1016/j.meegid.2012.08.012

Hall, T.A., 1999, Bioedit: A user-friendly biological sequence alignment editor and analysis program for windows $95 / 98 / N t$, Nucleic Acids Symposium Series, $\mathrm{pp}$ 95-98, Oxford University Press, Oxford.

Hecker, Y.P., Moore, D.P., Manazza, J.A., Unzaga, J.M., Späth, E.J., Pardini, L.L. et al., 2013, 'First report of seroprevalence of Toxoplasma gondii and Neospora caninum in dairy sheep from Humid Pampa, Argentina', Tropical Animal Health and Production 45, 1645-1647. http://dx.doi.org/10.1007/s11250-013-0396-1

Hemphill, A., Vonlaufen, N. \& Naguleswaran, A., 2006, 'Cellular and immunological basis of the host-parasite relationship during infection with Neospora caninum', Parasitology 133, 261-278. http://dx.doi.org/10.1017/\$0031182006000485

Howe, L., Collett, M., Pattison, R., Marshall, J., West, D. \& Pomroy, W., 2012, 'Potential involvement of Neospora caninum in naturally occurring ovine abortions in New Zealand', Veterinary Parasitology 185, 64-71. http://dx.doi.org/10.1016/j. vetpar.2011.10.033

Howe, L., West, D., Collett, M., Tattersfield, G., Pattison, R., Pomroy, W. et al., 2008, 'The role of Neospora caninum in three cases of unexplained ewe abortions in the Southern North Island of New Zealand', Small Ruminant Research 75, 115-122. http://dx.doi.org/10.1016/j.smallrumres.2007.08.001

Hughes, J., Williams, R., Morley, E., Cook, D., Terry, R., Murphy, R. et al., 2006, 'The prevalence of Neospora caninum and co-infection with Toxoplasma gondii by PCR analysis in naturally occurring mammal populations', Parasitology 132, 29-36. $\mathrm{http}: / /$ dx.doi.org/10.1017/S0031182005008784 
Jolley, W., Mcallister, M., Mcguire, A. \& Wills, R., 1999, 'Repetitive abortion in Neospora-infected ewes', Veterinary Parasitology 82, 251-257. http://dx.doi.org/ 10.1016/S0304-4017(99)00017-5

Kaufmann, H., Yamage, M., Roditi, I., Dobbelaere, D., Dubey, J., Holmdahl, O. et al., 1996, 'Discrimination of Neospora caninum from Toxoplasma gondii and other apicomplexan parasites by hybridization and PCR', Molecular and Cellular Probes 10, 289-297. http://dx.doi.org/10.1006/mcpr.1996.0038

Larkin, M.A., Blackshields, G., Brown, N., Chenna, R., Mcgettigan, P.A., Mcwilliam, H. et al., 2007, 'Clustal W and clustal X version 2.0', Bioinformatics 23, 2947-2948. http://dx.doi.org/10.1093/bioinformatics/btm404

Liu, Z.-K., Li, J.-Y. \& Pan, H., 2015, 'Seroprevalence and risk factors of Toxoplasma gondii and Neospora caninum infections in small ruminants in China', Preventive Veterinary Medicine 118, 488-492. http://dx.doi.org/10.1016/j prevetmed.2014.12.017

Magaña, A., Sánchez, F., Villa, K., Rivera, L. \& Morales, E., 2015, 'Systemic neosporosis in a dog treated for immune-mediated thrombocytopenia and hemolytic anemia', Veterinary Clinical Pathology 44, 592-596. http://dx.doi.org/10.1111/vcp.12287

Mazuz, M.L., Shkap, V., Wollkomirsky, R., Leibovich, B., Savitsky, I., Fleiderovitz, L. et al., 2016, 'Neospora caninum: Chronic and congenital infection in consecutive pregnancies of mice', Veterinary Parasitology 219, 66-70. http://dx.doi. pregnancies of mice', Veterinary
org/10.1016/j.vetpar.2016.01.013

Moreno, B., Collantes-Fernández, E., Villa, A., Navarro, A., Regidor-Cerrillo, J. \& Ortega-Mora, L., 2012, 'Occurrence of Neospora caninum and Toxoplasma gondii infections in ovine and caprine abortions', Veterinary Parasitol 187, 312-318. http://dx.doi.org/10.1016/j.vetpar.2011.12.034

Muller, N., Zimmermann, V., Hentrich, B. \& Gottstein, B., 1996, 'Diagnosis of Neospora caninum and Toxoplasma gondii infection by PCR and DNA hybridization immunoassay', Journal Clinical Microbiology 34, 2850-2852.

Nasir, A., Ashraf, M., Khan, M., Javeed, A., Yaqub, T., Avais, M. et al., 2012, 'Prevalence of Neospora caninum antibodies in sheep and goats in Pakistan', Journa Parasitology 98, 213-215. http://dx.doi.org/10.1645/GE-2863.1

Şuteu, O., Paştiu, A., Györke, A., Avram, E. \& Cozma, V., 2013, 'Molecular detection of Neospora caninum in slaughtered goat kids from Romania', Science Parasitology 14, 43-46.

Pabón, M., López-Gatius, F., García-Ispierto, I., Bech-Sabat, G., Nogareda, C. \& Almería, S., 2007, 'Chronic Neospora caninum infection and repeat abortion in dairy cows: A 3-year study', Veterinary Parasitology 147, 40-46. http://dx.doi.org/10.1016/j. vetpar.2007.03.017
Paula, V., Rodrigues, A., Richtzenhain, L., Cortez, A., Soares, R. \& Gennari, S., 2004 'Evaluation of a PCR based on primers to NC5 gene for the detection of Neospora caninum in brain tissues of bovine aborted fetuses', Veterinary Research Communications 28, 581-585. http://dx.doi.org/10.1023/B:VERC.0000042877. Communicat
07684.89

Pena, H., Soares, R., Ragozo, A., Monteiro, R., Yai, L., Nishi, S. et al., 2007, 'Isolation and molecular detection of Neospora caninum from naturally infected sheep from Brazil', Veterinary Parasitology 147, 61-66. http://dx.doi.org/10.1016/j. Brazil', Veterinary
vetpar.2007.03.002

Reichel, M.P. Alejandra Ayanegui-Alcerreca, M, Gondim, LF. \& Ellis, JT., 2013, 'What is the global economic impact of Neospora caninum in cattle-the billion dollar question', International Journal for Parasitology 43, 133-142. http://dx.doi.org/ question', International Journal

Rettigner, C., De Meerschman, F., Focant, C., Vanderplasschen, A. \& Losson, B., 2004 'The vertical transmission following the reactivation of a Neospora caninum chronic infection does not seem to be due to an alteration of the systemic immune response in pregnant cba/ca mice', Parasitology 128, 149-160. http://dx.doi.org/ response in pregnant cba/ca mice
$10.1017 / \mathrm{S} 0031182003004402$

Santos, S.L, De Souza Costa, K., Gondim, L.Q, Da Silva, M.S.A Uzeda, R.S., AbeSandes, K. \& Gondim, L.F.P., 2010, 'Investigation of Neospora caninum, Sandes, K. \& Gondim, L.F.P., 2010, Investigation of Neospora caninum,
Hammondia sp., and Toxoplasma gondii in tissues from slaughtered beef cattle in Hahia, Brazil', Parasitology Research 106, 457-461. http://dx.doi.org/10.1007/ Bahia, Brazil', Parasitol
s00436-009-1686-4

Sasani, F., Javanbakht, J., Seifori, P., Fathi, S. \& Hassan, M.A., 2013, 'Neospora caninum as causative agent of ovine encephalitis in Iran', Pathology Discovery 1, 5. http://dx.doi.org/10.7243/2052-7896-1-5

Silva, M.S., Uzeda, R.S., Costa, K.S., Santos, S.L., Macedo, A.C., Abe-Sandes, K. \& Gondim, L.F.P., 2009, 'Detection of Hammondia Heydorni and related coccidia (Neospora caninum and Toxoplasma gondii) in goats slaughtered in Bahia, Brazil', Veterinary Parasitology 162, 156-159. http://dx.doi.org/10.1016/j.vetpar.2009. 02.007

Tamura, K., Stecher, G., Peterson, D., Filipski, A. \& Kumar, S., 2013, 'Mega6: Molecula evolutionary genetics analysis version 6.0', Molecular Biology and Evolution 30 2725-2729. http://dx.doi.org/10.1093/molbev/mst197

Yamage, M., Flechtner, O. \& Gottstein, B., 1996, 'Neospora caninum: Specific oligonucleotide primers for the detection of Brain "Cyst" dna of experimentally infected nude mice by the polymerase chain reaction (PCR)', Journal Parasitology 82, 272-279. http://dx.doi.org/10.2307/3284160 


\section{Appendix 1}

EF581827

GU194965

KF649844

EU073599

kp702735

KR106185

kp702736

DQ077662

HM031966

KR106182

KR106183

DQ077661

KF649848

kp 702737

DQ132438

KR106181

KF649846

EF202080

GU194963

GU194964

GU194961

GU194962

KR106184

DQ0 077663

DQ132437

EU073600

KF64984 7

EF581827

GU194965

KF649844

EU073599

kp702735

KR106185

kp702736

DQ077662

HM031966

KR106182

KR106183

DQ077661

KF649848

kp702737

DQ132438

KR106181

KF649846

EF202080

GU194963

GU194964

GU194961

GU194962

KR106184

DQ077663

DQ132437

EU073600

KF649847
CAGTCAACCTACGTCTTCTGCCCCTTCCCTCGTCCGCTTGCTCCCTATGCATAATCTCCC 60 CAGTCAACCTACGTCTTCTGCCCCTTCCCTCGTCCGCTTGCTCCCTATGCATAATCTCCC 60 CAGTCAACCTACGTCTTCTGCCCCTTCCCTCGTCCGCTTGCTCCCTATGCATAATCTCCC 60 CAGTCAACCTACGTCTTCTGCCCCTTCCCTCGTCCGCTTGCTCCCTATGCATAATCTCCC 60 CAGTCAACCTACGTCTTCTGCCCCTTCCCTCGTCCGCTTGCTCCCTATGCATAATCTCCC 60 CAGTCAACCTACGTCTTCTGCCCCTTCCCTCGTCCGCTTGCTCCCTATGCATAATCTCCC 60 CAGTCAACCTACGTCTTCTGCCCCTTCCCTCGTCCGCTTGCTCCCTATGCATAATCTCCC 60 CAGTCAACCTACGTCTTCTGCCCCTTCCCTCGTCCGCTTGCTCCCCATGCATAATCTCCC 60 CAGTCAACCTACGTCTTCTGCCCCTTCCCTCGTCCGCTTGCTCCCTATGCATAATCTCCC 60 CAGTCAACCTACGTCTTCTGCCCCTTCCCTCGTCCGCTTGCTCCCTATGCATAATCTCCA 60 CAGTCAACCTACGTCTTCTGCCCCTTCCCTCGTCCGCTTGCTCCCTATGCATAATCTCCA 60 CAGTCAACCTACGTCTTCTGCCCCTTCCCTCGTCCGCTTGCTCCCTATGCATAATCTCCC 60 CAGTCAACCTACGTCTTCTGCCCCTTCCCTCGTCCGCTTGCTCCCTATGCATAATCTCCC 60 CAGTCAACCTACGTCTTCTGCCCCTTCCCTCGTCCGCTTGCTCCCTATGCATAATCTCCC 60 CAGTCAACCTACGTCTTCTGCCCCTTCCCTCGTCCGCTTGCTCCCTATGAATAATCTCCC 60 CAGTCAACCTACGTCTTCTGCCCCTTCCCTCGTCCGCTTGCTCCCTATGCATAATCTCCC 60 CAGTCAACCTACGTCTTCTGCCCCTTCCCTCGTCCGCTTGCTCCCTATGCATAATCTCCC 60 CAGTCAACCTACGTCTTCTGCCCCTTCCCTCGTCCGCTTGCTCCCTATGCATAATCTCCA 60 CAGTCAACCTACGTCTTCTGCCCCTTCCCTCGTCCGCTTGCTCCCTATGCATAATCTCCC 60 CAGTCAACCTACGTCTTCTGCCCCTTCCCTCGTCCGCTTGCTCCCTATGCATAATCTCCC 60 CAGTCAACCTACGTCTTCTGCCCCTTCCCTCGTCCGCTTGCTCCCTATGCATAATCTCCC 60 CAGTCAACCTACGTCTTCTGCCCCTTCCCTCGTCCGCTTGCTCCCTATGCATAATCTCCC 60 CAGTCAACCTACGTCTTCTGCCCCTTCCCTCGTCCGCTTGCTCCCTATGCATAATCTCCC 60 CAGTCAACCTACGTCTTCTGCTCCTCCCCTCGTCCGCTTGCTCCCTATGCATAATCTCCC 60 CAGTCAACCTACGTCTTCTGCCCCTTCCCTCGTCCGCTTGCTCCCTATGAATAATCTCCC 60 CAGTCAACCTACGTCTTCTGCCCCTTCCCTCGTCCGCTTGCTCCCTATGCATAATCTCCC 60 CAGTCAACCTACGTCTTCTGCCCCTTCCCTCGTCCGCTTGCTCCCTATGCATAATCTCCC 60 $* * * * * * * * * * * * * * * * * * * * * . * * * . * * * * * * * * * * * * * * * * * * * . * * * . * * * * * * * * *$.

CCGTCATCAGTGCCGCCGGTGTTGCCTCAACACAGAACACTAAACTCTGGATAAGTATCA 120 CCGTCATCAGTGCCGCCGGTGTTGCCTCAACACAGAACACTAAACTCTGGATAAGTATCA 120 CCGTCATCAGTGCCGCCGGTGTTGCCTCAACACAGAACACTGAACTCTGGATAAGTATCA 120 CCGTCATCAGTGCCGCCGGTGTTGCCTCAACACAGAACACTGAACTCTGGATAAGTATCA 120 CCGTCATCAGTGCCGCCGGTGTTGCCTCAACACAGAACACTGAACTCTĞGATAAGTATCA 120 CCGCCATCAGTGCCGCCGGTGTTGCA-CAACACAGAACACTGAACTCTGGATAAGTATCA 119 CCGC̄CATCAGTGCCGCCGGTGTTGCA-CAACACAGAACACTGAACTCTĞGATAAGTATCA 119 CCGCCATCAGTGCCGCCGGTGTTGCCTCAACACAGAACACTGAACTCTGGATAAGTATCA 120 CCGC̄CATCAGTGCCGCCGGTGTTGCCTCAACACAGAACACTGAACTCTḠGATAAGTATCA 120 CCGTCATCAGTGCCGCCGGTGTTGCCTCAACACAGAACACTGAACTCTGGATAAGTATCA 120 GCGTCATCAGTGCCGCCGGTGTTGCCTCAACACAGAACACTGAACTCTGGGATAAGTATCA 120 CCGTCATCAGTGCCGCCGGTGTTGCCTCAACACAGAACACTGAACTCTG̈GATAAGTATCA 120 CCGTCATCAGTGCCGCCGGTGTTGCCTCAACACAGAACACTGAACTCTGGATAAGTATCA 120 CCGTCATCAGTGCCGCCGGTGTTGCCTCAACACAGAACACTGAACTCTCGGTAAGTATCA 120 CCGTCATCAGTGCCGCCGGTGTTGCCTCAACACAGAACACTGAACTCTCGATAAGTATCA 120 CCGTCATCAGCGCCGCCGGTGTTGCCTCAACACAGAACACTGAACTCTCGATAAGTATTA 120 CCGTCATCAGC̄GCCGCCGGTGTTGCCTCAACACAGAACACTGAACTCTC̄GATAAGTATTA 120 CCGTCATCAGTGCCGCCGGTGTTGCCTCAACACAGAACACTGAACTCTGGATAAGTATTA 120 CCGTCATCAGCGCCGCCGGTGTTGCCCCAACACAGAACACTGAACTCTCGGTAAGTATCA 120 CCGTCATCAGCGCCGCCGGTGTTGCCCCAACACAGAACACTGAACTCTCGGTAAGTATCA 120 CCGTCATCAGC̄GCCGCCGGTGTTGCCCCAACACAGAACACTGAACTCTCGG̈TAAGTATCA 120 CCGTCATCAGCGCCGCCGGTGTTGCCCCAACACAGAACACTGAACTCTCGGTAAGTATCA 120 CCGTCATCAGC̄GCCGCCGGTGTTGCCTCAACACAGAACACTGAACTCTC̄GĀTAAGTATCA 120 CTGTCATCAGTGCCGCCGGTGTTGCCTCAACACAGGACACTGAACTCTCGATAAGTATCA 120 CTGTCATCAGTGCCGCCGGTGTTGCCTCAACACAGĀACACTGAACTCTCGATAAGTATCA 120 CCGTCATCAGTGCCGCCGGTGTTGCCTCAACACAGAACACTGAACTCTCGATAAGTATCA 120 CCGTCATCAGTGCCGCCGGTGTTGCCTCAACACAGAACACTGAACTCTCGATAAGTATCA 120

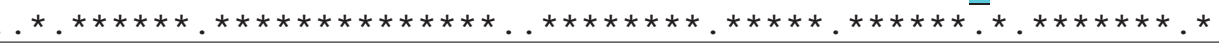

Our sheep samples are represented in red (accession nos. KR106181, KR106182, KR106183, KR106184). An asterisk represents an exact match between all sequences; a dot (•) represents a mismatch in at least one sequence.

FIGURE 1-A1: Sequence alignment of Nc-5 gene of Neospora caninum from mammalian and bird hosts. 


\section{Appendix 1 (Continues...).}

EF581827

GU194965

KF649844

EU073599

kp702735

KR106185

kp702736

DQ077662

HM031966

KR106182

KR106183

DQ077661

KF649848

$\mathrm{kp} 702737$

DQ132438

KR106181

KF649846

EF202080

GU194963

GU194964

GU194961

GU194962

KR106184

$\mathrm{DQ} 077663$

DQ132437

EU073600

KF649847

EF581827

GU194965

KF649844

EU073599

$\mathrm{kp} 702735$

KR106185

kp702736

DQ077662

HM0 31966

KR106182

KR106183

DQ0 77661

KF649848

kp702737

DQ132438

KR106181

KF649846

EF 202080

GU194963

GU194964

GU194961

GU194962

KR106184

DQ0 77663

DQ132437

EU073600

KF649847
TTGACACATTCTCCACTCCCTGACGCAGGCTCATTTCAACGTGACGAATGACTAACCACA 180 TTGACACATTCTCCACTCCCTGACGCAGGCTCATTTCAACGTGACGAATGACTAACCACA 180 TTGACACACTGTCCACACCCTGACGCAGGCTCATTTCAACGTGACGAATGACTAACCACA 180 TTGACACACTGTCCACACCCTGACGCAGGCTCATTTCAACGTGACGAATGACTAACCACA 180 TTGACACACTGTCCACACCCTGACGCAGGCTCATTTCAACGTGACGAATGACTAACCACA 180 TTGACACACTGTCCACACCCTGACGCAGGCTCATTTCAACGTGACGAATGACTAACCACA 179 TTGACACACTGTCCACACCCTGACGCAGGCTCATTTCAACGTGACGAATGACTAACCACA 179 TTGACACACTGTCCACACCCTGACGCAGGCTCATTTCAACGTGACGAATGACTAACCACA 180 TTGACACACTGTCCACACCCTGACGCAGGCTCATTTCAACGTGACGAATGACTAACCACA 180 TTGACACACTGTCCACACCCTGACGCAGGCTCATGTCAACGTGACGAATGACTAACCACA 180 TTGACACACTGTCCACAGCCTGACGCAGGCTCATGTCAACGTGACGAATGACTAACCACA 180 TTGACACACTGTCCACA ČCCTGACGCAGGCTGATTTCAACGTGACGAATGACTAACCGCA 180 TTGACACACTGTCCACACCCTGACGCAGGCTGATTTCAACGTGACGAATGACTAACCĀCA 180 TTGACACACTGTCCACACCCTGACGCAGGCTCATTTCAACGTGACGAATGACTAACCACA 180 TTGACACACTGTCCACACCCTGACGCGGGCTCATTTCAACGTGACGAATGACTAACCACA 180 TTGGCACACTGTCCACACCCTGACGCÄGGCTGATTTCAACGTGACGAATGACTAACCACA 180 TTGḠCACACTGTCCACACCCTGACGCAGGCTGATTTCAACGTGACGAATGACTAACCACA 180 TTGĞACACTGTCCACACACTGACGCAGGCTGATTTCAACGTGACGAATGACTAACCACA 180 TTGA AACACTGTCCTCACCCTGACGCAGGATGATTTCAACGTGACGAATGACTAACCACA 180 TTGACACACTGTCCTCACCCTGACGCAGGATGATTTCAACGTGACGAATGACTAACCACA 180 TTGACACACTGTCCTCACCCTGACGCAGGCTGATTTCAACGTGACGAATGACTAACCACA 180 TTGACACACTGTCCTCACCCTGACGCAGGCTGATTTCAACGTGACGAATGACTAACCACA 180 TTGACACACTGTCCTCACCCTGACGCAGGCTGATTTCAACGTGACGAATGACTAACCACA 180 TTGACACACTGTCCACACCCTGACGCAGGCTGATTTCAACGTGACGAATGACTAACCACA 180 TTGACACACTGTCCACACCCTGACGCAGGCTGATTTCAACGTGACGAATGACTAACCACA 180 TTGACACACTGTCCACACCCTGACGCAGGCTGATTTCAACGTGACGAATGACTAACCACA 180 TTGACACACTGTCCACACCCTGACGCAGGCTGATTTCAACGTGACGAATGACTAACCACA 180

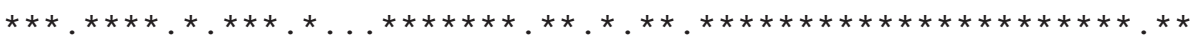

AACCACGTATCCCACCTCTCACCGCTACCAACTCCCTCGGTTCACCC 227 AACCACGTATCCCACCTCTCACCGCTACAGACTCCCTCGGTTCACCC 227 AACCACGTATCCCACCTCTCACCGCTACCAACTCCCTCGGTTCACCC 227 AACCACGTATCCCACCTCTCACCGCTACCAACTCCCTCGGTTCACCC 227 AACCACGTATCCCACCTCTCACCGCTACCAACTCCCTCGGTTCACCC 227 AACCACGTATCCCACCTCTCACCGCTACCAACTCCCTCGGTTCACCC 226 AACCACGTATCCCACCTCTCACCGCTACCAACTCCCTCGGTTCACCC 226 AACCACGTATCCCACCTCTCACCGCTACCAACTCCCTCGGTTCACCC 227 AACCACGTATCCCACCTCTCACCGCTACCAACTCCCTCGGTTCACCC 227 AACCACGTATCCCACCTCTCACCGCTACCAACTCCCTCGGTTCACCC 227 AACCACGTATCCCACCTCTCACCGCTACCAACTCCCTCGGTTCACCC 227 AACCACGCATCCCACCTCTCACCGCTACCAACTCCCTCGGTTCACCC 227 AACCACGTATCCCACCTCTCACCGCTACCAACTCCCTCGGTTCACCC 227 AACCACGTATCCCACCTCTCACCGCTACCAACTCCCTCGGTTCACCC 227 AACCACGTATCCCACCTCTCACCGCTACCAACTCCCTCGGTTCACCC 227 AACCACGTATCCCACCTCTCACCGCTACCAACTCCCTCGGTTCACCC 227 AACCACGTATCCCACCTCTCACCGCTACCAACTCCCTCGGTTCACCC 227 AACCACGTATCCCACCTCTCACCGCTACCAACTCCCTCGGTTCACCC 227 AACCACGTATCCCACCTCTCACCGCTACCAACTCCCTCGGTTCACCC 227 AACCACGTATCCCACCTCTCACCGCTACCAACTCCCTCGGTTCACCC 227 AACCACGTATCCCACCTCTCACCGCTACCAACTCCCTCGGTTCACCC 227 AACCACGTATCCCACCTCTCACCGCTACCAACTCCCTCGGTTCACCC 227 AACCACGTATCCCACCTCTCACCGCTACCAACTCCCTCGGTTCACCC 227 AACCACGTATCCCACCTCTCACCGCTACCAACTCCCTCGGTTCACCC 227 AACCACGTATCCCACCTCTCACCGCTACCAACTCCCTCGGTTCACCC 227 AACCACGTATCCCACCTCTCACCGCTACCAACTCCCTCGGTTCACCC 227 AACCACGTATCCCACCTCTCACCGCTACCAACTCCCTCGGTTCACCC 227

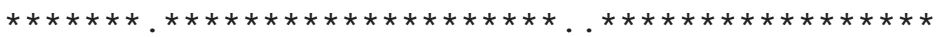

Our sheep samples are represented in red (accession nos. KR106181, KR106182, KR106183, KR106184). An asterisk represents an exact match between all sequences; a dot (•) represents a mismatch in at least one sequence.

FIGURE 1-A1 (Continues...): Sequence alignment of Nc-5 gene of Neospora caninum from mammalian and bird hosts. 